\title{
Zero-resistance States Induced by Bichromatic Microwaves
}

\author{
Alejandro Kunold* \\ Departamento de Ciencias Básicas, Universidad Autónoma Metropolitana-Azcapotzalco, \\ Av. San Pablo 180, México D. F. 02200, México \\ Manuel Torres ${ }^{\dagger}$ \\ Instituto de Física, Universidad Nacional Autónoma de México, \\ Apartado Postal 20-364, México Distrito Federal 01000, México
}

\begin{abstract}
We have studied the bichromatic photoresistance states of a two dimensional electron gas in the regime of microwave induced resistance oscillations. Zudov and coworkers ${ }^{1}$ found clear experimental evidence of zero-resistance states by measuring the bichromatic resistance in a bidimensional gas of electrons. They found that the bichromatic resistance closely replicates the superposition of the two monochromatic components provided that both contributions are positive. However, the superposition principle is no longer valid if one of the two contributions give rise to a zero-resistance state. The experiments by Zudov and coworkers confirm that negative resistance states are rapidly driven into zero-resistance states through A. V. Andreev's symmetry breaking. In this work we present a model for the bichromatic-photoconductivity of a two dimensional electron system subjected to a uniform magnetic field. Our model includes both components of the microwave radiation, a uniform magnetic field and impurity scattering effects. The conductivity is calculated from a Kubo-like formula. Our calculations reproduce the main features of Zudov's experimental results.
\end{abstract}

\section{INTRODUCTION.}

Recently, two experimental groups ${ }^{2.3}$, reported the existence of zero-photoresistance states (ZRS) in ultraclean twodimensional electron systems (2DES) subjected to microwave (MW) radiation and moderate perpendicular magnetic fields.

In these experiments the microwave-induced resistance oscillations (MIRO) exhibit periods proportional to the inverse of the magnetic field alternated with ZRS regions governed by the ratio $\epsilon=\omega / \omega_{c}$, where $\omega_{c}$ and $\omega$ are the cyclotron an MW frequency. The oscillation amplitudes reach maxima at $\epsilon=\omega / \omega_{c}=j$ and minima at $\epsilon=j+1 / 2$, for $j$ an integer ${ }^{2}$.

Despite the large amount of theoretical work ${ }^{4.5 .6 .7 .8 .9 .12}$ the origin of ZRS remains elusive. Nevertheless most theories agree that some mechanism produces negative-resistance states (NRS) that rapidly drive the system into a ZRS due to Andreev's instabilities 12 . Some of the models ${ }^{4,5,8}$ suggest that the main cause of NRS is photon-assisted scattering by impurities or disorder. Alternative explanations $\frac{9}{-}$ propose that NRS arise from MW induced distribution function fluctuations. It is important to mention that some theories do not need to invoke Andreev's instabilities ${ }^{10.11}$.

In recent experiments 1 the bichromatic resistance was found to be well described by the superposition of the two independent monochromatic components in the following terms. When both monochromatic resistances are positive or zero the bichromatic resistivity agrees with the sum of both components. If only one of the monochromatic resistances is zero the bichromatic resistance is lower than the expected from the direct superposition principle.

In this work we develop a model that is based on the exact solution of the Shrödinger equation of a $2 \mathrm{D}$ electron in the presence of a static magnetic field $B$ interacting with a MW radiation and a perturbative calculation of randomly distributed impurities. For small radiation intensities the conductivity follows the superposition principle suggested by Zudov ${ }^{1}$. The lowering of the bichromatic resistivities is shown to originate from the superposition of NRS and positive resistance (PR) from the independent monochromatic contributions.

\section{THE MODEL}

In order to calculate the expectation value of the current density we need the time dependent density matrix $\rho$ which obeys the von Neumann equation $i \partial \rho / \partial t=\left[H+V_{\text {ext }}, \rho\right]$ where $V_{\text {ext }}$ is the bias voltage and the Hamiltonian is

\footnotetext{
* Corresponding author: e-mail: akb@correo.azc.uam.mx

$\dagger$ e-mail: torres@fisica.unam.mx
} 

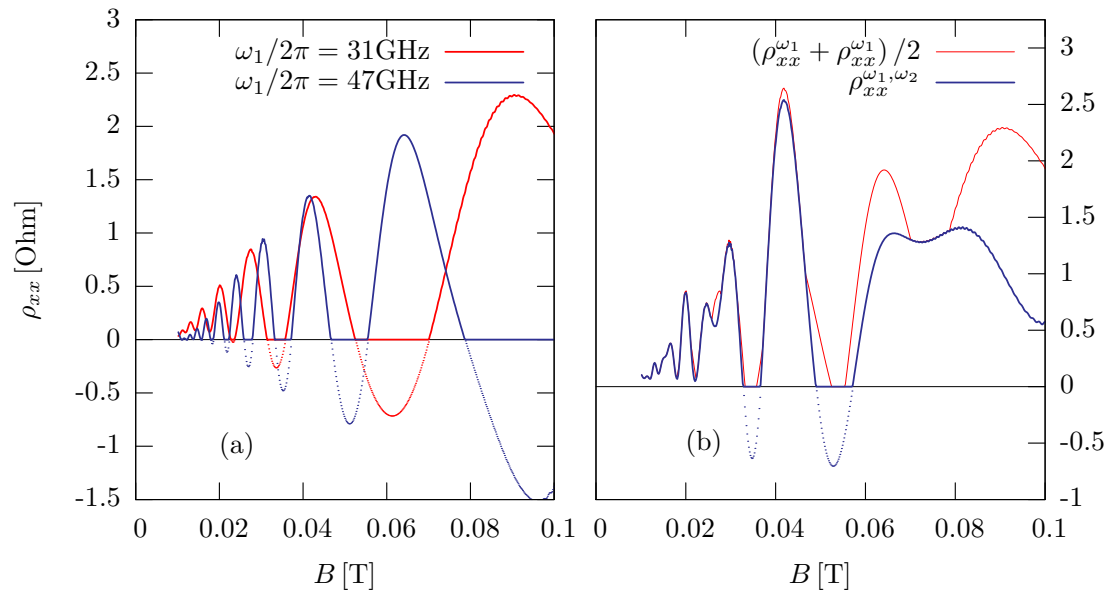

FIG. 1: Panel (a) represents the resistivity under monochromatic radiation for $\omega_{1} / 2 \pi=31 \mathrm{GHz}$ (blue) and $\omega_{2} / 2 \pi=47 \mathrm{GHz}$ (red). In panel (b) the blue line shows the bichromatic conductivity $\rho_{x x}$ and the red line is a plot of $\rho_{x x 1}+\rho_{x x 2}$. The dotted lines indicates NRS. In this example $E_{1}=220 \mathrm{~V} / \mathrm{m}, E_{2}=350 \mathrm{~V} / \mathrm{m}$. The inset contains plots of successive values of $E_{2}=350$, $400,450,500,550 \mathrm{~V} / \mathrm{m}$.

given by

$$
H=H_{\left\{B, \omega_{1}, \omega_{2}\right\}}+V(\boldsymbol{r}) .
$$

The MW bichromatic radiation's electric field $\boldsymbol{E}=\boldsymbol{E}_{1}+\boldsymbol{E}_{2}=E_{x} \boldsymbol{i}+E_{y} \boldsymbol{j}$ is obtained from the vector potential $\boldsymbol{A}(t)=$ $-\frac{1}{2} \boldsymbol{r} \times \boldsymbol{B}+\operatorname{Re}\left[\frac{\boldsymbol{E}_{1}}{\omega_{1}} \exp \left(i \omega_{1} t\right)+\frac{\boldsymbol{E}_{2}}{\omega_{2}} \exp \left(i \omega_{2} t\right)\right]$. Here, the electric fields $\boldsymbol{E}_{1}$ and $\boldsymbol{E}_{2}$ are decomposed in their polarization vectors $\epsilon_{x 1}, \epsilon_{y 1}$ and $\epsilon_{x 2}, \epsilon_{y 2}$ respectively. The unperturbed Hamiltonian contains both components of the microwave radiation and a uniform magnetic field

$$
\begin{aligned}
H_{\left\{B, \omega_{1}, \omega_{2}\right\}} & =\frac{1}{2 m^{*}}(\boldsymbol{p}+e \boldsymbol{A})^{2}+e \boldsymbol{E} \cdot \boldsymbol{x} \\
& =\frac{1}{2 m^{*}}\left(Q_{1}^{2}+P_{1}^{2}\right)+e E_{x}(t)\left(Q_{1}-P_{2}\right)+e E_{y}(t)\left(Q_{2}-P_{1}\right),
\end{aligned}
$$

where

$$
\begin{array}{ll}
P_{1}=p_{x}-\frac{e B}{2} y-\frac{e}{2} \int^{t} d t^{\prime} E_{x}\left(t^{\prime}\right), & P_{2}=Q_{1}-e B x \\
Q_{1}=p_{y}+\frac{e B}{2} x-\frac{e}{2} \int^{t} d t^{\prime} E_{y}\left(t^{\prime}\right), & Q_{2}=P_{1}+e B y
\end{array}
$$

are canonical operators that obey the commutation relations $\left[Q_{1}, P_{1}\right]=\left[Q_{2}, P_{2}\right]=i e B,\left[Q_{1}, Q_{2}\right]=\left[P_{1}, P_{2}\right]$ $=\left[Q_{1}, P_{2}\right]=\left[Q_{2}, P_{1}\right]=0$. The impurity scattering potential is given by

$$
V(\boldsymbol{r})=\sum_{i} \int \frac{d^{2} q}{(2 \pi)^{2}} V(q) \exp \left[i \boldsymbol{q} \cdot\left(\boldsymbol{r}-\boldsymbol{r}_{i}\right)\right] .
$$

where $m^{*}$ is the effective mass of the electron, $\boldsymbol{r}_{i}$ the position of the $i$ th impurity and the explicit form of $V(q)$ depends on the type of impurity. For the sake of simplicity in this work we will only consider uncharged impurities.

The unperturbed Hamiltonian $H_{\left\{B, \omega_{1}, \omega_{2}\right\}}$ can be reduced to a harmonic oscillator through a unitary transformation given by $\underline{\underline{13}}$

$$
W(t)=\exp \left(i \eta_{1} Q_{1}\right) \exp \left(i \zeta_{1} P_{1}\right) \exp \left(i \eta_{2} Q_{2}\right) \exp \left(i \zeta_{2} P_{2}\right) \exp \left(-i \int^{t} d t^{\prime} \mathcal{L}\left(t^{\prime}\right)\right)
$$

where the functions $\eta_{i}(t)$ and $\zeta_{i}(t)$ are solutions of the dynamical equations that follow form the variation of the classical Lagrangian $\mathcal{L}=\frac{\omega_{c}}{2}\left(\eta_{1}^{2}+\zeta_{1}^{2}\right)+\dot{\zeta}_{1} \eta_{1}+\dot{\zeta}_{2} \eta_{2}+e l_{B}\left[E_{x}\left(\zeta_{1}+\eta_{2}\right)+E_{y}\left(\eta_{1}+\zeta_{2}\right)\right]$ with $l_{B}=\sqrt{\hbar / e B}$ the magnetic 
longitude. The solution to the classical dynamical equations and the unitary transformation (6) constitute the exact solution for the unperturbed Hamiltonian. The impurity potential is considered through first order time dependent perturbation theory. Solving von Neumann equation in the linear approximation, the longitudinal conductivity is calculated as described in ${ }^{13}$ leading to expressions for the dark $\sigma_{x x}^{D}$ and illuminated $\sigma_{x x}^{\omega_{1}, \omega_{2}}$ conductivities. The dark part of the conductivity leads to Shubnikov-de Haas oscillations. The illuminated longitudinal conductivity is given by

$$
\sigma_{x x}^{\omega_{1}, \omega_{2}}=\frac{e^{2}}{\pi \hbar} \int d \mathcal{E} \sum_{\mu \nu} \sum_{l_{1} l_{2}} \operatorname{Im} G_{\mu}(\mathcal{E}) B^{l_{1} l_{2}}\left(\mathcal{E}, \mathcal{E}_{\nu}\right)\left|\Delta_{\mu \nu}^{l_{1} l_{2}(x)}\right|^{2} .
$$

The temperature dependence enters the conductivity through

$$
B^{l_{1} l_{2}}\left(\mathcal{E}, \mathcal{E}_{\nu}\right)=-\frac{\partial}{\partial \mathcal{E}_{0}}\left\{\left[f\left(\mathcal{E}+\omega_{1} l_{1}+\omega_{2} l_{2}+\mathcal{E}_{0}\right)-f(\mathcal{E})\right] G_{\nu}\left(\mathcal{E}+\omega_{1} l_{1}+\omega_{2} l_{2}+\mathcal{E}_{0}\right)\right\}
$$

where $f$ is the Fermi distribution function, $G_{\nu}(\mathcal{E})=1 /(\mathcal{E}-i \Gamma)$,

$$
\begin{gathered}
\rho_{1}^{(j)}=\frac{e l_{B} E_{1}}{2} \frac{\omega_{c} \epsilon_{y j}-i \omega_{j} \epsilon_{x j}}{\omega_{j}^{2}-\omega_{c}^{2}+i \omega_{j} \Gamma}, \quad \rho_{2}^{(j)}=\frac{e l_{B} E_{2}}{2} \frac{\omega_{c} \epsilon_{x j}+i \omega_{j} \epsilon_{y j}}{\omega_{j}^{2}-\omega_{c}^{2}+i \omega_{j} \Gamma} \\
\Delta_{i}=\frac{e \omega_{c} l_{B}^{2} E_{i}}{\omega_{i}\left(\omega_{i}^{2}-\omega_{c}^{2}-i \omega_{i} \Gamma\right)}\left[\omega_{i}\left(q_{x} \epsilon_{x i}+q_{y} \epsilon_{y i}\right)-i \omega_{c}\left(q_{x} \epsilon_{y i}-q_{y} \epsilon_{x i}\right)\right]
\end{gathered}
$$

and $\Gamma=2 \pi e / m \mu_{0}$ is a phenomenological damping factor. The term

$$
\begin{aligned}
\left|\Delta_{\mu \nu}^{l_{1} l_{2}(i)}\right|^{2}=\delta_{\mu \nu}[ & \left.\left|\rho_{i}^{(1)}\right|^{2}\left(\delta_{l_{1}, 1}+\delta_{l_{1},-1}\right) \delta_{l_{2}, 0}+\left|\rho_{i}^{(2)}\right|^{2}\left(\delta_{l_{2}, 1}+\delta_{l_{2},-1}\right) \delta_{l_{1}, 0}\right] \\
& +\frac{1}{2}\left|\frac{a_{i} C_{\mu \nu}^{l_{1} l_{2}+}}{\mathcal{E}_{\mu \nu}+\omega_{1} l_{1}+\omega_{2} l_{2}-\omega_{c}}+\frac{b_{i} C_{\mu \nu}^{l_{1} l_{2}-}}{\mathcal{E}_{\mu \nu}+\omega_{1} l_{1}+\omega_{2} l_{2}+\omega_{c}}\right|
\end{aligned}
$$

accounts for the MIRO where $a_{x}=b_{x}=1$ and $a_{y}=-b_{y}=-i$ and

$$
\begin{array}{r}
C_{\mu \nu}^{l_{1} l_{2} \pm}=\sqrt{\frac{\mu !}{\nu !}}\left(\frac{l_{B}}{\sqrt{2}}\right)^{\nu-\mu+1} \sum_{i} \int \frac{d^{2} q}{(2 \pi)^{2}} V(q) e^{-i \boldsymbol{q} \cdot \boldsymbol{r}_{i}-\left(l_{B} q\right)^{2} / 4}\left(q_{x}+i q_{y}\right)^{\nu-\mu+1}\left(\mp q_{y}-q_{x}\right) \\
L_{\mu}^{\nu-\mu}\left(\frac{l_{B}^{2} q^{2}}{2}\right)\left(\frac{\Delta_{1}}{\left|\Delta_{1}\right|}\right)^{l_{1}}\left(\frac{\Delta_{2}}{\left|\Delta_{2}\right|}\right)^{l_{2}}\left[J_{l_{1}}\left(\left|\Delta_{1}\right|\right) J_{l_{2}}\left(\left|\Delta_{2}\right|\right)-\delta_{l_{1}, 0} \delta_{l_{2}, 0}\right]
\end{array}
$$

with $L_{\mu}^{\nu-\mu}\left(l_{B}^{2} q^{2} / 2\right)$ the associated Laguerre polynomials.

\section{SUPERPOSITION PRINCIPLE}

In most experiments $\frac{1}{1}$ the intensity of bichromatic radiation is small compared to the separation of the Landau levels, that is $l_{B} e E_{1,2} / \hbar \omega_{c} \ll 1$, and the wave length of the microwave radiation is larger than the magnetic length $l_{B}$ thus $q l_{B} \ll 1$ and $\left|\Delta_{1}\right|,\left|\Delta_{2}\right| \ll 1$. Thus, the two leading terms of the illuminated longitudinal conductivity under this conditions are $\sigma_{x x}^{\omega_{1}, \omega_{2}}=\left(\sigma_{x x}^{\omega_{1}}+\sigma_{x x}^{\omega_{2}}\right) / 2$, where $\sigma_{x x}^{\omega_{1}}$ and $\sigma_{x x}^{\omega_{2}}$ are the corresponding monochromatic conductivities given by

$$
\begin{gathered}
\sigma_{x x}^{\omega_{1}}=\frac{e^{2}}{\pi \hbar} \int d \mathcal{E} \sum_{\mu \nu} \operatorname{Im} G_{\mu}(\mathcal{E}) B^{1,0}\left(\mathcal{E}, \mathcal{E}_{\nu}\right)\left|\Delta_{\mu \nu}^{1,0(x)}\right|^{2} \\
\sigma_{x x}^{\omega_{2}}=\frac{e^{2}}{\pi \hbar} \int d \mathcal{E} \sum_{\mu \nu} \operatorname{Im} G_{\mu}(\mathcal{E}) B^{0,1}\left(\mathcal{E}, \mathcal{E}_{\nu}\right)\left|\Delta_{\mu \nu}^{0,1(x)}\right|^{2} .
\end{gathered}
$$

Given the complexity of the previous expressions, the integrals over the energy where performed numerically. The bichromatic magnetoresistivity is then

$$
\rho_{x x}^{\omega_{1}, \omega_{2}}=\frac{\sigma_{x x}^{\omega_{1}, \omega_{2}}}{\left(\sigma_{x x}^{\omega_{1}, \omega_{2}}\right)^{2}+\sigma_{x y}^{2}} \approx \frac{\sigma_{x x}^{\omega_{1}, \omega_{2}}}{\sigma_{x y}^{2}}=\frac{\sigma_{x x}^{\omega_{1}}+\sigma_{x x}^{\omega_{2}}}{2 \sigma_{x y}^{2}} \approx \frac{\rho_{x x}^{\omega_{1}}+\rho_{x x}^{\omega_{2}}}{2} .
$$


In Fig. 1 we observe the monochromatic components of the magneto-resistivity [panel (a)] for frequencies $\omega_{1} / 2 \pi=$ $31 \mathrm{GHz}, \omega_{2} / 2 \pi=47 \mathrm{GHz}$, the mobility is $\mu=2 \times 10^{3} \mathrm{~m}^{2} / \mathrm{Vs}$ and $\Gamma=34.1 \times 10^{-6} \mathrm{eV}$. The NRS are plotted with dotted lines. The bichromatic magneto-resistivity is plotted in panel (b). We notice that when two positive resistivities superimpose the bichromatic resistivity is simply their sum. More specifically, at $B=0.04 \mathrm{~T}$ the peaks from the monochromatic contributions overlap. On the other hand, when a positive resistivity is superimposed with a ZRS, the bichromatic resistivity is suppressed indicating the existence of NRS. At $B=0.06 \mathrm{~T}$, for example, $\rho_{x x}^{\omega_{1}}>0$ and $\rho_{x x}^{\omega_{2}}<0$ thus $\rho_{x x}^{\omega_{1}, \omega_{2}}<\rho_{x x}^{\omega_{1}}+\rho_{x x}^{\omega_{2}}$. In the inset of panel (b) we observe that as $E_{2}$ arises, it is even possible to produce ZRS (for $E_{2}=500$ and $550 \mathrm{~V} / \mathrm{m}$ ) by overlapping a NRS with a PR state.

\section{CONCLUSIONS}

Based on linear response theory we have presented a theoretical model to explain the MIRO superposition principle observed by Zudov. We have shown that the interaction of impurity assisted NRS an PR states from the monochromatic components are responsible for the enhancement and suppression of the bichromatic resistivity. Our calculations on the bichromatic conductivity have similar features as those observed by Zudov.

We acknowledge financial support by CONACyT J 43110-F, G 32736-E, and UNAM project No. IN113305.

1 M.A. Zudov, R.R. Du, L.N. Pfeiffer, and K.W. West Phys. Rev. Lett. 96, 236804 (2006).

2 M. A. Zudov, R. R. Du, J. A. Simmons, J. L. Reno Phys. Rev. B 64, 201311(R) (2001); M. A. Zudov, R. R. Du, L. N. Pfeiffer, K. W. West, Phys. Rev. Lett 90, 046807 (2003); M. A. Zudov, Phys. Rev. B 69, 041304(R) (2004).

${ }^{3}$ R. G. Mani, J. H. Smet, K. von klitzing, V. Narayanamurti, W.b. Johnson, Umansky, Nature 420, 646 (2002); Phys. Rev. Lett 92, 146801 (2004); R. G. Mani, Physica E 22, 1 (2004).

4 V. I. Ryzhii, R. Suris, J Phys. Cond. Matt. 15, 6855 (2003).

5 A. C. Durst, S. Sachdev, N. Read, S. M. Girvin, Phys. Rev. Lett. 91, 086803 (2003).

6 J. Shi, X. C. Xie, Phys. Rev. Lett. 91, 086801 (2003).

7 X. L. lei, S. Y. Liu, Phys. Rev. Lett. 91, 226805 (2003).

8 M. G. Vavilov, I. L. Aleiner, Phys. Rev. B 69, 035303 (2004).

9 I. A. Dmitriev, A. D. Mirlin, D. G. Polyakov Phys. Rev. Lett. 91, 226802 (2003); I. A. Dmitriev, M. G. Vavilov, I. L. Aleiner, A. D. Mirlin, D. G. Polyakov, cond-mat/0310668 (2003); cond-mat/0409590 (2004).

10 D. -H Lee and J. M. Leinaas, Phys. Rev. B 69, 115336 (2004).

11 J. Iñarrea and G. Platero, Phys. Rev. Lett. 94, 016806 (2005).

12 A. V. Andreev, I. L. Aleiner, A. J. Millis, Phys. Rev. Lett. 91, 056803 (2003).

13 Manuel Torres, Alejandro Kunold Phys. Rev. B 71, 115313 (2005). 